\title{
Planejamento Fatorial para a Investigação das Propriedades Espectroscópicas, Efeitos Relativísticos e de Solvatação de Derivados Halogenados da 2-Amino-1,4-Naftoquinona
}

\author{
Eduardo P. Rocha, Lívia C. T. Lacerda, Mateus A. Gonçalves, Maíra S. Pires, \\ Telles C. Silva, Henrique A. Rodrigues \& Teodorico C. Ramalho
}

\section{Introdução}

Nos últimos anos, o interesse por processos fotoquímicos e fotofísicos tem aumentado. ${ }^{1}$ Muitos desses processos apresentam aplicações industriais, como a fotocatálise, ou aplicações biomédicas, como as sondas fluorescentes para o diagnóstico de imagem. ${ }^{2}$ Muitos compostos apresentam características fluorescentes, dentre esses compostos, encontram-se os derivados de naftoquinonas. ${ }^{3}$ Esses compostos, de origem natural, apresentam diversas aplicações biológicas, como antitumorais, antifúngica, antimalárica, entre outras. ${ }^{4}$ Derivados de naftoquinonas já foram testados por grupos de pesquisa3 como sondas fluorescentes em alguns tipos de câncer, como o câncer de cólon e de boca., ${ }^{3,5}$ Estudos das propriedades desses derivados apresentam, dessa forma, grande importância, uma vez que alterações estruturais podem fornecer sondas fluorescentes mais eficientes, seletivas e com um maior precisão. ${ }^{1}$

Modificações estruturais, como adição de halogênios em determinadas posições da molécula pode promover alterações das propriedades espectroscópicas, o que possibilita a geração de novas regiões de absorção e emissões. ${ }^{6}$ Estudos de derivados halogenados de naftoquinonas empregam a espectroscopia de fluorescência ${ }^{7}$, Raman $^{8}$, UV-VIS $^{9}$, entre outras análises para a avaliação dos padrões de emissão e absorção desses compostos, bem como, por estudos teóricos, que envolvem a análise das energias de absorção, de emissão, a análise da diferença entre os orbitais homo-lumo, entre outras características. ${ }^{7}$
Recentes estudos teóricos envolvem, também, a análise dos diferentes mecanismos de emissão de fluorescência apresentado por esses compostos. ${ }^{1,10}$

Dentre os estudos teóricos existem diferentes métodos que permitem a análise de processos de absorção e emissão, como os métodos Multi-Referenciais CASSCF, CASPT2, entre outros ${ }^{9}$. Nos últimos anos, o emprego da teoria do funcional de Densidade (DFT) tem se tornado importante por permitir a análise de diferentes sistemas químicos com tamanhos diferenciados, como para a análise de complexos metálicos e sistemas biológicos. ${ }^{11} \mathrm{~A}$ análise de propriedades de absorção e emissão é realizada com o emprego da Teoria do Funcional de Densidade dependente do tempo (TDDFT), que permite a análise das transições verticais de compostos diversos, bem como a análise da diferença entre os orbitais homo-lumo e pode ser aplicada aos estudos dos mecanismos de fluorescência de inúmeras moléculas. ${ }^{12}$

Dentre os diversos aspectos relacionados ao processo de emissão e absorção, os efeitos relativísticos têm sido considerados importantes para o estudo de átomos com alta massa molecular, como o Bromo e o Iodo. ${ }^{13}$ Esses efeitos têm sido amplamente estudados para átomos como o ouro13, podendo ser negligenciados em compostos orgânicos que envolvam apenas átomos com baixa massa molecular. Entretanto, efeitos relativísticos, como o acoplamento spin-órbita, que está relacionado ao desdobramento dos níveis de energia de átomos pesados, podem influenciar significativamente propriedades eletrônicas de compostos químicos. ${ }^{14}$

Desde o enunciado da Equação de Dirac para resolução 
dos efeitos relativísticos, diferentes métodos têm sido desenvolvidos, como o método de Douglas-Kroll-Hess, que transforma o hamiltoniano de quatro componentes para duas componentes apenas. ${ }^{15-17} \mathrm{Ou}$ o método da Aproximação Regular de Ordem Zero (ZORA), que emprega o método de Pauli para a resolução da Equação de Dirac para compostos químicos. ${ }^{18,19}$ Esses métodos modificam o hamiltoniano da Equação de Schröedinger e promovem o cálculo de propriedades relativísticas para sistemas químicos que apresentam grande aplicação a estudos de propriedades espectroscópicas de complexos metálicos e estruturas químicas que envolvam átomos de alto peso molecular. ${ }^{20,21}$

Desta forma, para compostos com átomos pesados, a incorporação de efeitos relativísticos é essencial para o cálculo acurado de propriedades espectroscópicas. Todavia, a escolha de qual o melhor método relativístico a ser aplicado normalmente requer a avaliação de vários fatores. Dentre esses fatores, encontra-se, por exemplo, a utilização ou não do acoplamento spin-órbita e o emprego de funções de base relativísticas. Neste contexto, o planejamento experimental empregando a metodologia de planejamento fatorial pode auxiliar na escolha das melhores condições para o estudo. O planejamento fatorial é uma abordagem estatística que envolve a construção de uma combinação de fatores, escolhidos dentre os parâmetros que se deseja estudar, para a investigação da significância desses fatores sobre o estudo. Existem diferentes formas de construir o planejamento fatorial $(2 \mathrm{~K}, 3 \mathrm{~K}, \ldots)$, e com isso diferentes formas de modelar o sistema na busca pela interpretação correta dos resultados.

O presente estudo visa a aplicação da metodologia de planejamento fatorial do tipo $3 \mathrm{~K}$ para avaliação de métodos relativísticos sobre o processo de absorção de compostos halogenados de 2-amino-1,4-naftoquinona visando avaliar as melhores condições e os fatores significativos para o estudo da propriedade espectroscópica dos derivados halogenados.

\section{Metodologia}

Otrabalho foidesenvolvidoaplicandoométodoTD-DFT/ B3LYP ${ }^{22}$ para o cálculo das propriedades espectroscópicas dos compostos 2-amino-3-Bromo-1,4-Naftoquinona $(\mathrm{ABNQ})^{23}$ e 2-amino-3-Iodo-1,4-Naftoquinona (AINQ), conforme estruturas químicas apresentadas na Figura 1. Os 8 primeiros estados excitados foram avaliados com o programa $\mathrm{ORCA}^{24}$ empregando o método $\mathrm{COSMO}^{7,19}$ para simular o efeito do solvente metanol, ${ }^{23}$ o qual foi empregado como solvente nos estudos experimentais para o composto ABNQ. O método de Tamm-Dancoff (TDA) ${ }^{25}$ foi empregado para simular o efeito de relaxação do solvente no estado excitado dentro do método TD-DFT.
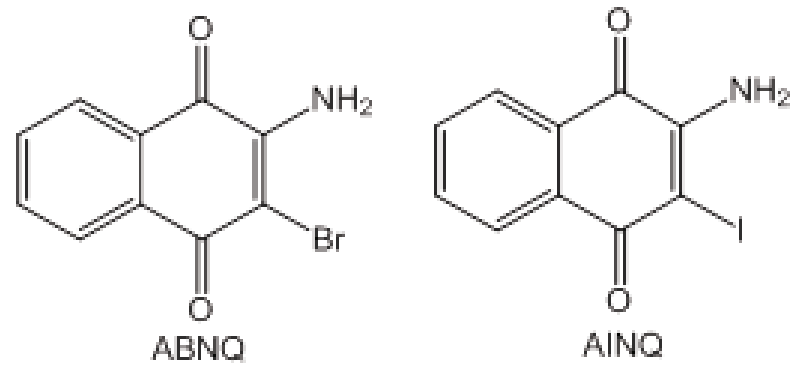

Figura 1. Estrutura química dos compostos halogenados da 3-amino1,4-naftoquinona.

O planejamento fatorial foi executado dentro do programa Statistica $\AA$, empregando metodologia tipo $3 \mathrm{~K}$, conforme descrito na Tabela 1. Os parâmetros do planejamento fatorial foram a presença do acoplamento spin-órbita (sem o acoplamento, o acoplamento spinórbita apenas utilizando a carga nuclear efetiva (SO (1)) e o acoplamento spin-órbita com aproximação de campo e potencial efetivo (SO (3))), a função de onda (TVZ, TVZ_ZORA e TVZ_DKH) ${ }^{24}$ implementada no programa ORCA para avaliação das funções de onda relativísticas e o hamiltoniano (não relativístico, ZORA e DKH2). ${ }^{26} \mathrm{~A}$ variável resposta para o composto $\mathrm{ABNQ}$ foi a diferença entre o valor calculado e o valor experimental ${ }^{23}$, já para o composto AINQ a variável resposta foi apenas o valor calculado, uma vez que para esse composto não há valor experimental reportado na literatura é o presente momento. A estimativa dos efeitos foram realizados com o auxílio do programa Statistica ${ }^{\circledR}$ e descritos na Tabela 2, bem como a análise ANOVA, descrita na Tabela 3. Para critério de aceitação foi empregado, juntamente com a ANOVA, o Teste t a $5 \%$ de probabilidade. 


\section{Resultados e Discussão}

O planejamento Fatorial do tipo $3 \mathrm{~K}$ para o composto 2-amino-3-Bromo-1,4-Naftoquinona (ABNQ) seguiu os parâmetros estipulados na Tabela 1. Nesse planejamento, avaliou-se no parâmetro A o efeito do acoplamento spin-órbita, no parâmetro $\mathrm{B}$ a função de base e no parâmetro $\mathrm{C}$ o método relativístico. Os níveis de cada parâmetro foram estipulados desde o método sem efeitos relativísticos (sem o acoplamento spin-orbita, função de base não relativística e o hamiltoniano não relativístico), passando pelo nível médio (adição do efeito spinórbita, função de base com implementação ZORA, e método ZORA), até o nível alto (adição do efeito spin-órbita, função de base com implementação DKH e método DKH2). Avaliouse, também, a interação dos fatores entre si. Como resposta, os valores de absorção empregando o método TD-DFT e o modelo COSMO, para simular o efeito do solvente metanol, foram calculados. $\mathrm{O}$ valor experimenta ${ }^{23}$ foi comparado aos ${ }^{27}$ ensaios calculados e representados na Tabela 1.

Tabela 1. Planejamento Fatorial do tipo $3^{3}$ com os níveis e fatores avaliados no planejamento.

\begin{tabular}{|c|c|c|c|c|}
\hline Nível & -1 & $\mathbf{0}$ & +1 & \\
\hline $\begin{array}{l}\text { (A) Acoplamento Spin- } \\
\text { Órbita }\end{array}$ & Sem & $\mathrm{SO}(1)$ & $\mathrm{SO}(3)$ & \\
\hline (B) Função de base & TVZ & TVZ_ZORA & TVZ_DKH & \\
\hline (C) Método Relativístico & Não-Rel. & ZORA & DKH2 & \\
\hline $\mathbf{A}$ & B & $\mathbf{C}$ & R (Metanol) ABNQ & R(Metanol) AINQ \\
\hline-1 & -1 & -1 & $458,7(+6,7)^{*}$ & 461 \\
\hline-1 & -1 & 0 & $457,2(+5,2)$ & 460,8 \\
\hline-1 & -1 & 1 & $457,7(+5,7)$ & 462,9 \\
\hline-1 & 0 & -1 & $457,1(+5,1)$ & 463,2 \\
\hline-1 & 0 & 0 & $458,4(+6,4)$ & 460,8 \\
\hline-1 & 0 & 1 & $454,2(+2,2)$ & 460,6 \\
\hline-1 & 1 & -1 & $459,4(+7,4)$ & 463,4 \\
\hline-1 & 1 & 0 & $459,4(+7,4)$ & 461 \\
\hline-1 & 1 & 1 & $452,7(+0,7)$ & 460,6 \\
\hline 0 & -1 & -1 & $458,7(+6,7)$ & 461 \\
\hline 0 & -1 & 0 & $457,2(+5,2)$ & 460,8 \\
\hline 0 & -1 & 1 & $457,7(+5,7)$ & 462,9 \\
\hline 0 & 0 & -1 & $457,1(+5,1)$ & 463,2 \\
\hline 0 & 0 & 0 & $458,4(+6,4)$ & 460,8 \\
\hline 0 & 0 & 1 & $454,2(+2,2)$ & 460,6 \\
\hline 0 & 1 & -1 & $459,4(+7,4)$ & 463,4 \\
\hline 0 & 1 & 0 & $459,4(+7,4)$ & 461 \\
\hline 0 & 1 & 1 & $452,7(+0,7)$ & 460,6 \\
\hline 1 & -1 & -1 & $458,7(+6,7)$ & 461 \\
\hline 1 & -1 & 0 & $457,2(+5,2)$ & 460,8 \\
\hline 1 & -1 & 1 & $457,7(+5,7)$ & 462,9 \\
\hline 1 & 0 & -1 & $457,1(+5,1)$ & 463,2 \\
\hline 1 & 0 & 0 & $458,4(+6,4)$ & 460,8 \\
\hline 1 & 0 & 1 & $454,2(+2,2)$ & 460,6 \\
\hline 1 & 1 & -1 & $459,4(+7,4)$ & 463,4 \\
\hline 1 & 1 & 0 & $459,4(+7,4)$ & 461 \\
\hline 1 & 1 & 1 & $452,7(+0,7)$ & 460,6 \\
\hline
\end{tabular}

* Valores em parênteses são referentes ao erro comparado ao valor experimental de $452 \mathrm{~nm}^{23}$ 
O modelo quadrático empregado para a análise do planejamento fatorial possui a forma descrita na Equação 1. O planejamento fatorial deste estudo busca encontrar os melhores fatores em relação aos parâmetros que gerem os menores erros, buscando uma minimização dos resultados. Pode-se notar que os fatores que se mostraram significativos foram com relação ao fator $\mathrm{C}$, ou seja, ao método relativístico adotado. E a interação entre a função de base e o método relativístico também se mostraram importantes para o composto avaliado.

As Equações 1 e 2 são geradas pelos valores significativos descritos na Tabela 2 e 3, para os compostos ABNQ e AINQ, respectivamente. Para o ABNQ, a equação é referente ao erro absoluto gerado pela comparação do valor calculado com o experimental. Para o composto AINQ, a equação foi gerada com o valor bruto do comprimento de onda obtido pelo cálculo. A análise da Equação 1, que representa o modelo quadrático para o fatorial $3^{\mathrm{K}}$, mostrou que o fator $\mathrm{A}$ não é significativo para a construção das respostas, ou seja, a adição do acoplamento spin-órbita para o composto ABNQ. O fator B, apesar de estar dentro do modelo, também não foi significativo para o estudo. Dessa forma, as funções de base estudada, com a adição dos efeitos relativísticos, não produziram efeitos sobre a resposta. O parâmetro C foi significativo para o estudo, destacando um valor de $-3,53 \%$ para a análise linear, o que indica que ao passar do nível baixo (-1) para o nível médio ou alto $(0,+1)$ a resposta diminui em $3,53 \%$.

Tabela 2 - Estimativa dos efeitos para o modelo quadrático do planejamento fatorial $3^{3}$ para o composto ABNQ baseado nos valores de Erro.

\begin{tabular}{|c|c|c|c|c|c|c|}
\hline Fator & Efeito & Erro padrão & $\mathbf{t}(\mathbf{1 7 )}$ & $\mathbf{p}$ & $\begin{array}{c}\mathbf{- 9 5 \%} \text { Limite de } \\
\text { confiança }\end{array}$ & $\begin{array}{c}\text { +95\% Limite de } \\
\text { Confiança }\end{array}$ \\
\hline Média & 5,20 & 0,22 & 23,16 & 0,00 & 4,72 & 5,67 \\
\hline A & 0,00 & 0,54 & 0,00 & 1,00 & $-1,16$ & 1,16 \\
\hline A2 & 0,00 & 0,47 & 0,00 & 1,00 & $-1,00$ & 1,00 \\
\hline B & $-0,70$ & 0,54 & $-1,27$ & 0,22 & $-1,86$ & 0,46 \\
\hline B2 & $-0,95$ & 0,47 & $-1,99$ & 0,06 & $-1,95$ & 0,05 \\
\hline C & $-3,53$ & 0,54 & $-6,42$ & 0,00 & $-4,69$ & $-2,37$ \\
\hline C2 & $+1,7$ & 0,47 & 3,56 & 0,00 & 0,69 & 2,70 \\
\hline $\mathrm{A} * \mathrm{~B}$ & 0,00 & 0,67 & 0,00 & 1,00 & $-1,42$ & 1,42 \\
\hline $\mathrm{A}$ C & 0,00 & 0,67 & 0,00 & 1,00 & $-1,42$ & 1,42 \\
\hline $\mathrm{B} * \mathrm{C}$ & $-2,85$ & 0,67 & $-4,23$ & 0,00 & $-4,27$ & $-1,42$ \\
\hline
\end{tabular}

De acordo com o modelo, a variação do hamiltoniano sem efeito relativístico para o método de Douglas-Krolln-Hess de segunda ordem (DKH2) diminuiu o erro estimado para a resposta. Isso significa que o método DKH2 se mostra mais acurado para o estudo dos valores de absorção e do comprimento de onda para a molécula ABNQ. A estimativa do efeito mostrou um valor de $-2,85 \%$ para a interação entre a função de base e o método relativístico, demonstrando que a resposta diminui quando passa do nível baixo para o médio ou alto em 2,85\%. Novamente, a união dos métodos sem os efeitos relativísticos gerou os maiores erros, indicando que a associação do método DKH2 com a função de base TZV_DKH é mais acurada no estudo do comprimento de onda e dos valores de absorção para a molécula ABNQ. Todos os valores calculados para a estimativa dos efeitos estão detalhados na Tabela 2 e na Tabela 3 apresenta-se a ANOVA do planejamento fatorial. 
Tabela 3. ANOVA para os planejamentos fatoriais dos compostos ABNQ e AINQ.

\begin{tabular}{|c|c|c|c|c|c|c|c|c|c|c|c|}
\hline Composto & & & ABNQ & & & & & & & & \\
\hline Fator & $\mathrm{SS}$ & $\mathrm{df}$ & MS & $\mathrm{F}$ & $\mathrm{P}$ & Fator & SS & $\mathrm{df}$ & MS & $\mathrm{F}$ & $\mathrm{P}$ \\
\hline A & 0,00 & 1 & 0,00 & 0,00 & 1,00 & A & 0,00 & 1 & 0,00 & 0,00 & 1,00 \\
\hline A2 & 0,00 & 1 & 0,00 & 0,00 & 1,00 & $\mathrm{~A}^{\wedge} 2$ & 0,00 & 1 & 0,00 & 0,00 & 1,00 \\
\hline B & 2,20 & 1 & 2,20 & 1,62 & 0,22 & B & 0,04 & 1 & 0,04 & 0,16 & 0,68 \\
\hline B2 & 5,41 & 1 & 5,41 & 3,97 & 0,06 & $\mathrm{~B}^{\wedge} 2$ & 0,04 & 1 & 0,04 & 0,15 & 0,70 \\
\hline $\mathrm{C}$ & 56,18 & 1 & 56,18 & 41,28 & 0,00 & $\mathrm{C}$ & 6,12 & 1 & 6,12 & 22,41 & 0,00 \\
\hline $\mathrm{C} 2$ & 17,34 & 1 & 17,34 & 12,74 & 0,00 & $\mathrm{C}^{\wedge} 2$ & 7,04 & 1 & 7,04 & 25,76 & 0,00 \\
\hline$A * B$ & 0,00 & 1 & 0,00 & 0,00 & 1,00 & $\mathrm{~A} * \mathrm{~B}$ & 0,00 & 1 & 0,00 & 0,00 & 1,00 \\
\hline $\mathrm{A} * \mathrm{C}$ & 0,00 & 1 & 0,00 & 0,00 & 1,00 & $\mathrm{~A}^{*} \mathrm{C}$ & 0,00 & 1 & 0,00 & 0,00 & 1,00 \\
\hline$B * C$ & 24,36 & 1 & 24,36 & 17,90 & 0,00 & $B * C$ & 16,56 & 1 & 16,56 & 60,62 & 0,00 \\
\hline Erro & 23,13 & 17 & 1,36 & - & - & Erro & 4,64 & 17 & 0,27 & - & - \\
\hline Total SS & 128,64 & 26 & - & - & - & Total SS & 34,46 & 26 & - & - & - \\
\hline
\end{tabular}

A análise fatorial empregando os valores calculados de comprimento de onda (Equação 2) ou o erro gerado ao se comparar com o valor experimental (Equação 1) produzem equações quadráticas semelhantes. Isto demonstra que nas duas ocasiões, o modelo se aplica, porém, ao se comparar ao valor experimental podese decidir melhor como interpretar cada modelo de acordo com o objetivo do estudo, que é de minimizar o erro em relação ao valor de comprimento de onda calculado.

$\mathrm{Y}=5,20-0,70 \mathrm{~B}-0,95 \mathrm{~B} 2-3,53 \mathrm{C}+1,70 \mathrm{C} 2-2,85 \mathrm{~B} * \mathrm{C}$

Equação 1

$\mathrm{Y}=457,2-0,70 \mathrm{~B}-0,95 \mathrm{~B} 2-3,53 \mathrm{C}+1,70 \mathrm{C} 2-2,85 \mathrm{~B} * \mathrm{C}$

Equação 2

A análise dos gráficos mostrados na Figura 2A e 2B apresentam todos os valores dos efeitos estimados para os dois modelos quadráticos e o valor de corte para o teste F a $0,5 \%$ de probabilidade, mostrando que os parâmetros são semelhantes e que os modelos se equiparam.

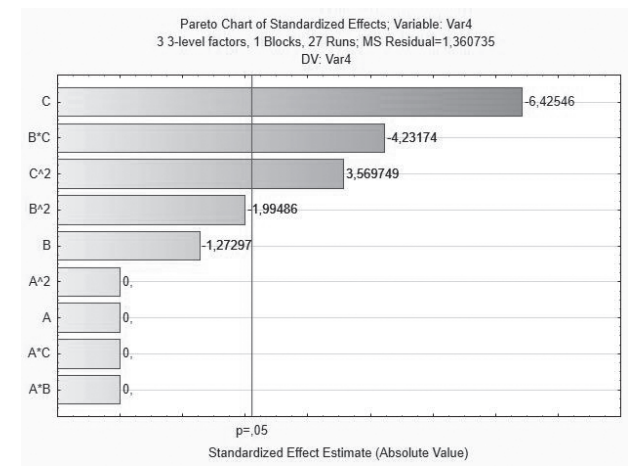

Figura 2A. Efeitos padronizados de Pareto para o planejamento fatorial $3 \mathrm{~K}$ baseado nos valores de comprimento de onda.

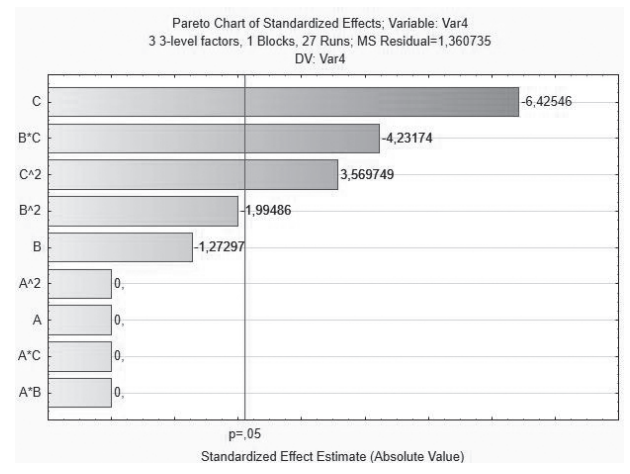

Figura 2B. Efeitos padronizados de Pareto para o planejamento fatorial $3 \mathrm{~K}$ baseado nos valores do erro. 
Baseado no modelo de planejamento fatorial apresentado para o ABNQ, pode-se construir um modelo semelhante para o 2-amino-3-Iodo-1,4-Naftoquinona (ver Figura 1) e comparar os dois modelos baseado na importância dos efeitos relativísticos para o cálculo de energia de absorção e do comprimento de onda desses compostos. Para o composto AINQ, os cálculos dos 27 ensaios foram realizados conforme descrito na Tabela 1. Os comprimentos de onda e a energia de absorção foram calculados empregando o método TDDFT juntamente com o modelo COSMO para simular o solvente metanol. Comparativamente ao composto ABNQ, o AINQ apresentou apenas o fator C como significativo, possuindo valor de $-1,16 \%$ enquanto o ABNQ apresentou o valor de $-3,53 \%$.

Tabela 4 - Estimativa dos efeitos para o modelo quadrático do planejamento fatorial 33 para o composto AINQ baseado nos valores de comprimento de onda.

\begin{tabular}{|c|c|c|c|c|c|c|}
\hline Fator & Efeito & Erro padrão & t(17) & P & $\begin{array}{c}\mathbf{- 9 5 \%} \text { Limite de } \\
\text { confiaça }\end{array}$ & $\begin{array}{c}\text { +95\% Limite de } \\
\text { Confiança }\end{array}$ \\
\hline Média & 461,58 & 0,10 & 4588,06 & 0,00 & 461,37 \\
\hline A & 0,00 & 0,24 & 0,00 & 1,00 & 461,80 \\
\hline A2 & 0,00 & 0,21 & 0,00 & 1,00 & $-0,51$ \\
\hline B & 0,10 & 0,24 & 0,40 & 0,68 & $-0,45$ & $-0,41$ \\
\hline B2 & $-0,08$ & 0,21 & $-0,39$ & 0,70 & $-0,53$ & 0,45 \\
\hline C & $-1,16$ & 0,24 & $-4,73$ & 0,00 & $-1,68$ \\
\hline C2 & $-1,08$ & 0,21 & $-5,07$ & 0,00 & $-1,53$ \\
\hline A*B & 0,00 & 0,30 & 0,00 & 1,00 & $-0,64$ \\
\hline A*C & 0,00 & 0,30 & 0,00 & 1,00 & $-0,63$ \\
\hline B*C & $-2,35$ & 0,30 & $-7,78$ & 0,00 & $-0,63$ \\
\hline
\end{tabular}

A análise das interações se mostrou significativa apenas entre os fatores $\mathrm{B}$ e C, mostrando valor de $-2,35 \%$ para o AINQ enquanto que para o ABNQ apresentou o valor de $-2,85 \%$, mostrando que para todos os efeitos que se mostraram significativos nesse estudo a resposta diminui quando sai do nível baixo (-1) para o nível médio $(0)$ ou alto $(+1)$, conforme descrito na Tabela 4. Novamente, pela análise da estimativa dos efeitos, pode-se observar que o método relativístico e a função de onda são significativos para estes estudos. O modelo apresentado para o composto ABNQ apresentou maior erro relativo ao valor experimental seguindo a orientação das estimativas dos efeitos, por comparação. O mesmo aconteceu com a estimativa dos efeitos para o AINQ, portanto, seguindo a mesma tendência, os fatores de menor nível apresentarão os maiores erros, ou seja, sem o acoplamento spin-órbita, com a função de base TVZ e com o hamiltoniano não relativístico. Assim, o melhor método para o estudo, ou seja, o método que apresenta os menores erros comparados ao valor experimental será, novamente, é o método de DKH2 e sua combinação com a função de base TZV_DKH.

\section{Conclusão}

O planejamento fatorial $3 \mathrm{k}$ para os compostos ABNQ e AINQ se mostrou satisfatório, revelando que para o cálculo TD-DFT do comprimento de onda e das energias de absorção utilizando o método COSMO para avaliar o solvente, apenas o hamiltoniano relativístico foi significativo para o cálculo, sendo o método DKH2 apresentou os menores erros. Mostrou, também, que na fase gás a função de onda é significativa para o cálculo. Em ambos os casos, a interação da função de onda com o método se mostrou significativo, sendo que o método DKH2 com a função de base TVZ_DKH geraram os menores erros para a propriedade observada. Com base no experimento avaliado para o ABNQ, pode ser desenvolvido método semelhante para o AINQ (ver Figura 1) comparando os parâmetros dos planejamentos fatoriais e definindo os melhores fatores para o cálculo das propriedades espectroscópicas. 


\section{Referências Bibliográficas}

1. Zhao, J.; Ji, S.; Chen, Y.; Guo, H.; Yang, P. 2012, 8803-8817.

2. Dugave, C.; Demange, L. 2003.

3. Laurieri, N.; Egleton, J. E.; Varney, A.; Thinnes, C. C.; Quevedo, C. E.; Seden, P. T.; Thompson, S.; Rodrigues-Lima, F.; Dairou, J.; Dupret, J. M.; Russell, A. J.; Sim, E. PLoS ONE 2013, 8.

4. Ferreira, V. F.; Jorqueira, A.; Souza, A. M. T.; Da Silva, M. N.; De Souza, M. C. B. V; Gouvêa, R. M.; Rodrigues, C. R.; Pinto, A. V.; Castro, H. C.; Santos, D. O.; Araújo, H. P.; Bourguignon, S. C. Bioorganic and Medicinal Chemistry 2006, 14, 5459-5466.

5. Luo, Y.; Li, Y.; Qiu, K. M.; Lu, X.; Fu, J.; Zhu, H. L. Bioorganic and Medicinal Chemistry 2011, 19, 6069-6076.

6. Bermejo-Bescós, P.; Martín-Aragón, S.; Jiménez-Aliaga, Karim L.BERMEJO-BESCÓS, P. et al. In vitro antiamyloidogenic properties of 1,4-naphthoquinones. Biochemical and Biophysical Research Communications, v. 400, n. 1, p. 169-174, 2010.; Ortega, A.; Molina, M. T.; Buxaderas, E.; Orellana, G.; Csákÿ, A. G. Biochemical and Biophysical Research Communications 2010, 400, 169-174.

7. Guzow, K.; Milewska, M.; Czaplewski, C.; Wiczk, W. Spectrochimica Acta - Part A: Molecular and Biomolecular Spectroscopy 2010, 75, 773-781.

8. Tucker, S. C.; Honn, K. V. Biochemical Pharmacology 2013, 85, 676-688.

9. Levine, B. G.; Mart, T. J. 2007.

10. Il'ichev, Y. V; Kühnle, W.; Zachariasse, K. a Journal of Physical Chemistry A 1998, 102, 5670-5680.

11. Alam, M. J.; Ahmad, S. Spectrochimica Acta Part A: Molecular and Biomolecular Spectroscopy 2012, 96, 992-1004.

12. Jana, S.; Dalapati, S.; Ghosh, S.; Guchhait, N. Journal of Photochemistry and Photobiology A: Chemistry 2013, 261, 31-40.

13. Gorin, D. J.; Toste, F. D. Nature 2007, 446, 395-403.

14. Arumugam, K.; Becker, U. Computational Redox Potential Predictions: Applications to Inorganic and Organic Aqueous Complexes, and Complexes Adsorbed to Mineral Surfaces; 2014; Vol. 4.

15. Kutzelnigg, W. Chemical Physics 1997, 225, 203-222.

16. Cheng, L.; Stopkowicz, S.; Gauss, J. International Journal of Quantum Chemistry 2014, 114, 1108-1127.

17. Bu, M.; Reimann, C.; Pantazis, D. A.; Bredow, T. 2008, 14491459.

18. Wolff, S. K.; Ziegler, T.; Van Lenthe, E.; Baerends, E. J. The Journal of Chemical Physics 1999, 110, 7689.

19. Green, T. F. G.; Yates, J. R. The Journal of chemical physics 2014, $140,234106$.
20. Vaara, J.; Pyykkö, P. Journal of Chemical Physics 2003, 118, 2973-2976

21. Pyykkö, P. Annual review of physical chemistry 2012, 63, 45-64.

22. Pyykkö, P. Angewandte Chemie (International ed. in English) 2004, 43, 4412-56.

23. Pushpam, S.; Kottaisamy, M.; Ramakrishnan, V. Spectrochimica Acta - Part A: Molecular and Biomolecular Spectroscopy 2013, $114,272-276$.

24. Neese, F. Wiley Interdisciplinary Reviews: Computational Molecular Science 2012, 2, 73-78.

25. Roemelt, M.; Beckwith, M. a; Duboc, C.; Collomb, M.-N.; Neese, F.; DeBeer, S. Inorganic chemistry 2012, 51, 680-7.

26. Haiduke, R. L. a.; Comar, M.; Da Silva, A. B. F. Chemical Physics 2006, 331, 173-177.

\section{Eduardo P. Rocha, Lívia C. T. Lacerda, Mateus A. Gonçalves, Maíra S. Pires, Telles C. Silva, Henrique A. Rodrigues \&Teodorico C. Ramalho*}

Departamento de Química, Universidade Federal de Lavras, Minas Gerais, Brasil.

*E-mail: teo@dqi.ufla.br 